\title{
10. Perinatal deaths
}

\section{Review of perinatal deaths 2009}

This chapter presents the results of perinatal death reviews carried out by the NSW Maternal and Perinatal Committee, which is a quality assurance committee established under the NSW Health Administration Act 1982. The Committee is privileged under the Act to carry out confidential reviews of maternal and perinatal deaths.

NSW Ministry of Health Policy Directive No. 2006_006 describes hospital procedures for review and reporting of perinatal deaths.' Since 2006, the Maternal and Perinatal Committee has carried out reviews of perinatal deaths occurring among fetuses or infants of at least 20 weeks gestation or at least 400 grams birth weight, bringing the Committee's review process in line with the criteria used by the NSW Perinatal Data Collection (PDC) since 2006 for reporting of births.

Perinatal deaths were reviewed by the Committee's Perinatal Outcomes Working Party. Both stillbirths and neonatal deaths were classified according to an obstetric cause-specific classification, the Perinatal Society of Australia and New Zealand Perinatal Death Classification (PSANZ-PDC). Neonatal deaths were also classified by neonatal cause according to the Perinatal Society of Australia and New Zealand Neonatal Death Classification (PSANZ-NDC). ${ }^{2}$
There were 838 perinatal deaths of at least 20 weeks gestation or at least 400 grams birth weight reported to the PDC in 2009. Confidential reports on 827 deaths were reviewed. Of the 598 stillbirths reported to the PDC, reviews were carried out on 580 (97.0\%). The PDC was notified of 240 neonatal deaths. Reviews were carried out on 247 neonatal deaths, which include neonatal deaths that occurred after discharge or transfer from the hospital of birth.

\section{Trends in causes of perinatal death}

Recent trends in causes of perinatal death are shown in Table 111. Over the period 2006 to 2009 the most common cause of perinatal death was congenital anomaly followed by unexplained antepartum death and spontaneous preterm birth. The percentage of deaths attributed to perinatal infection increased from $5.9 \%$ to $10.8 \%$ over the four-year period, while the percentage of deaths attributed to spontaneous preterm birth and specific perinatal conditions fell from $19.8 \%$ to $16.2 \%$ and 8.6 to $6.7 \%$ respectively.

\section{Table 111. Perinatal deaths by psanz-pdc classification, NSW 2006-2009\#}

\begin{tabular}{|c|c|c|c|c|c|c|c|c|}
\hline \multirow[t]{3}{*}{ PSANZ perinatal death classification } & & & \multicolumn{6}{|c|}{ Year } \\
\hline & \multicolumn{2}{|c|}{2006} & \multicolumn{2}{|c|}{2007} & \multicolumn{2}{|c|}{2008} & \multicolumn{2}{|c|}{2009} \\
\hline & No. & $\%$ & No. & $\%$ & No. & $\%$ & No. & $\%$ \\
\hline 1. Congenital anomaly & 194 & 22.5 & 189 & 21.2 & 192 & 23.2 & 198 & 23.9 \\
\hline 2. Perinatal infection & 51 & 5.9 & 62 & 7.0 & 93 & 11.2 & 89 & 10.8 \\
\hline 3. Hypertension & 26 & 3.0 & 34 & 3.8 & 42 & 5.1 & 29 & 3.5 \\
\hline 4. Antepartum haemorrhage & 71 & 8.2 & 73 & 8.2 & 74 & 8.9 & 67 & 8.1 \\
\hline 5. Maternal disease & 23 & 2.7 & 28 & 3.1 & 15 & 1.8 & 19 & 2.3 \\
\hline 6. Specific perinatal conditions & 74 & 8.6 & 53 & 5.9 & 48 & 5.8 & 55 & 6.7 \\
\hline 7. Hypoxic peripartum death & 27 & 3.1 & 40 & 4.5 & 30 & 3.6 & 26 & 3.1 \\
\hline 8. Fetal growth restriction & 53 & 6.1 & 35 & 3.9 & 22 & 2.7 & 19 & 2.3 \\
\hline 9. Spontaneous preterm & 171 & 19.8 & 156 & 17.5 & 121 & 14.6 & 134 & 16.2 \\
\hline 10. Unexplained antepartum death & 167 & 19.4 & 214 & 24.0 & 187 & 22.6 & 185 & 22.4 \\
\hline 11. No obstetric antecedent & 5 & 0.6 & 8 & 0.9 & 3 & 0.4 & 6 & 0.7 \\
\hline TOTAL & 862 & 100.0 & 892 & 100.0 & 827 & 100.0 & 827 & 100.0 \\
\hline
\end{tabular}


Table 112. Perinatal deaths by PSANZ-PDC classification and perinatal outcome, NSW 2009

\begin{tabular}{|c|c|c|c|c|c|c|c|}
\hline \multirow{3}{*}{\multicolumn{2}{|c|}{ PSANZ perinatal death classification }} & \multicolumn{6}{|c|}{ Perinatal outcome } \\
\hline & & \multicolumn{2}{|c|}{ Stillbirth } & \multicolumn{2}{|c|}{ Neonatal death } & \multicolumn{2}{|c|}{ TOTAL } \\
\hline & & No. & $\%$ & No. & $\%$ & No. & $\%$ \\
\hline \multirow[t]{14}{*}{1.} & Congenital abnormality & & & & & & \\
\hline & Central nervous system & 41 & 7.1 & 13 & 5.3 & 54 & 6.5 \\
\hline & Cardiovascular system & 19 & 3.3 & 4 & 1.6 & 23 & 2.8 \\
\hline & Urinary system & 11 & 1.9 & 4 & 1.6 & 15 & 1.8 \\
\hline & Gastrointestinal system & 0 & 0.0 & 2 & 0.8 & 2 & 0.2 \\
\hline & Chromosomal & 37 & 6.4 & 11 & 4.5 & 48 & 5.8 \\
\hline & Multiple & 24 & 4.1 & 8 & 3.2 & 32 & 3.9 \\
\hline & Musculoskeletal & 5 & 0.9 & 3 & 1.2 & 8 & 1.0 \\
\hline & Respiratory & 2 & 0.3 & 2 & 0.8 & 4 & 0.5 \\
\hline & Diaphragmatic hernia & 0 & 0.0 & 2 & 0.8 & 2 & 0.2 \\
\hline & Haematological & 1 & 0.2 & 0 & 0.0 & 1 & 0.1 \\
\hline & Tumours & 4 & 0.7 & 0 & 0.0 & 4 & 0.5 \\
\hline & Other specified & 4 & 0.7 & 1 & 0.4 & 5 & 0.6 \\
\hline & TOTAL & 148 & 25.5 & 50 & 20.2 & 198 & 23.9 \\
\hline \multirow[t]{12}{*}{2.} & Perinatal infection & & & & & & \\
\hline & Group B Streptococcus & 12 & 2.1 & 6 & 2.4 & 18 & 2.2 \\
\hline & E Coli & 7 & 1.2 & 2 & 0.8 & 9 & 1.1 \\
\hline & Listeria Monocytogenes & 0 & 0.0 & 1 & 0.4 & 1 & 0.1 \\
\hline & Spirochaetal & 1 & 0.2 & 0 & 0.0 & 1 & 0.1 \\
\hline & Other bacterial & 6 & 1.0 & 3 & 1.2 & 9 & 1.1 \\
\hline & Unspecified bacterial & 25 & 4.3 & 11 & 4.5 & 36 & 4.4 \\
\hline & Cytomegalovirus & 2 & 0.3 & 0 & 0.0 & 2 & 0.2 \\
\hline & Parvovirus & 4 & 0.7 & 0 & 0.0 & 4 & 0.5 \\
\hline & Protozoal eg Toxoplasma & 0 & 0.0 & 3 & 1.2 & 3 & 0.4 \\
\hline & Other unspecified organism & 4 & 0.7 & 2 & 0.8 & 6 & 0.7 \\
\hline & TOTAL & 61 & 10.5 & 28 & 11.3 & 89 & 10.8 \\
\hline \multirow[t]{7}{*}{3.} & Hypertension & & & & & & \\
\hline & Chronic: Essential & 2 & 0.3 & 1 & 0.4 & 3 & 0.4 \\
\hline & Gestational & 3 & 0.5 & 2 & 0.8 & 5 & 0.6 \\
\hline & Pre-eclampsia & 13 & 2.2 & 4 & 1.6 & 17 & 2.1 \\
\hline & Pre-eclampsia superimposed on chronic hypertension & 1 & 0.2 & 0 & 0.0 & 1 & 0.1 \\
\hline & Unspecified hypertension & 3 & 0.5 & 0 & 0.0 & 3 & 0.4 \\
\hline & TOTAL & 22 & 3.8 & 7 & 2.8 & 29 & 3.5 \\
\hline \multirow[t]{7}{*}{4.} & Antepartum haemorrhage & & & & & & \\
\hline & Placental abruption & 33 & 5.7 & 13 & 5.3 & 46 & 5.6 \\
\hline & Placenta praevia & 1 & 0.2 & 4 & 1.6 & 5 & 0.6 \\
\hline & Vasa praevia & 1 & 0.2 & 0 & 0.0 & 1 & 0.1 \\
\hline & Other & 4 & 0.7 & 1 & 0.4 & 5 & 0.6 \\
\hline & Undetermined origin & 6 & 1.0 & 4 & 1.6 & 10 & 1.2 \\
\hline & TOTAL & 45 & 7.8 & 22 & 8.9 & 67 & 8.1 \\
\hline \multirow[t]{6}{*}{5.} & Maternal disease & & & & & & \\
\hline & Diabetes/gestational diabetes & 6 & 1.0 & 2 & 0.8 & 8 & 1.0 \\
\hline & Maternal injury: Accidental & 1 & 0.2 & 0 & 0.0 & 1 & 0.1 \\
\hline & Lupus obstetric syndrome & 4 & 0.7 & 1 & 0.4 & 5 & 0.6 \\
\hline & Other specified & 4 & 0.7 & 1 & 0.4 & 5 & 0.6 \\
\hline & TOTAL & 15 & 2.6 & 4 & 1.6 & 19 & 2.3 \\
\hline \multirow[t]{9}{*}{6.} & Specific perinatal conditions & & & & & & \\
\hline & Twin-to-twin transfusion & 9 & 1.6 & 7 & 2.8 & 16 & 1.9 \\
\hline & Fetomaternal haemorrhage & 6 & 1.0 & 0 & 0.0 & 6 & 0.7 \\
\hline & Antepartum cord complications & 7 & 1.2 & 1 & 0.4 & 8 & 1.0 \\
\hline & Uterine abnormality & 2 & 0.3 & 10 & 4.0 & 12 & 1.5 \\
\hline & Alloimmune disease - Other & 0 & 0.0 & 1 & 0.4 & 1 & 0.1 \\
\hline & Idiopathic hydrops & 5 & 0.9 & 1 & 0.4 & 6 & 0.7 \\
\hline & Other & 6 & 1.0 & 0 & 0.0 & 6 & 0.7 \\
\hline & TOTAL & 35 & 6.0 & 20 & 8.1 & 55 & 6.7 \\
\hline
\end{tabular}




\begin{tabular}{|c|c|c|c|c|c|c|c|}
\hline \multirow{3}{*}{\multicolumn{2}{|c|}{ PSANZ perinatal death classification }} & \multicolumn{6}{|c|}{ Perinatal outcome } \\
\hline & & \multicolumn{2}{|c|}{ Stillbirth } & \multicolumn{2}{|c|}{ Neonatal death } & \multicolumn{2}{|c|}{ TOTAL } \\
\hline & & No. & $\%$ & No. & $\%$ & No. & $\%$ \\
\hline \multirow{9}{*}{7.} & Hypoxic peripartum death & & & & & & \\
\hline & Intrapartum complication - Uterine rupture & 0 & 0.0 & 2 & 0.8 & 2 & 0.2 \\
\hline & Intrapartum complication - Cord prolapse & 3 & 0.5 & 0 & 0.0 & 3 & 0.4 \\
\hline & Intrapartum complication - Shoulder dystocia & 1 & 0.2 & 1 & 0.4 & 2 & 0.2 \\
\hline & Intrapartum complication - Other & 0 & 0.0 & 3 & 1.2 & 3 & 0.4 \\
\hline & $\begin{array}{l}\text { Evidence of non-re-assuring fetal status in a normally } \\
\text { grown infant }\end{array}$ & 3 & 0.5 & 0 & 0.0 & 3 & 0.4 \\
\hline & $\begin{array}{l}\text { No intrapartum complications and no evidence of } \\
\text { non-reassuring fetal status }\end{array}$ & 0 & 0.0 & 1 & 0.4 & 1 & 0.1 \\
\hline & Unspecified & 7 & 1.2 & 5 & 2.0 & 12 & 1.5 \\
\hline & TOTAL & 14 & 2.4 & 12 & 4.9 & 26 & 3.1 \\
\hline \multirow[t]{7}{*}{8.} & Fetal growth restriction & & & & & & \\
\hline & $\begin{array}{l}\text { With evidence of reduced vascular perfusion on Doppler } \\
\text { studies and/or placental histopathology }\end{array}$ & 11 & 1.9 & 1 & 0.4 & 12 & 1.5 \\
\hline & No placental pathology & 1 & 0.2 & 0 & 0.0 & 1 & 0.1 \\
\hline & No examination of placenta & 1 & 0.2 & 2 & 0.8 & 3 & 0.4 \\
\hline & Other specified placental pathology & 1 & 0.2 & 0 & 0.0 & 1 & 0.1 \\
\hline & Unspecified or not known whether placental examined & 1 & 0.2 & 1 & 0.4 & 2 & 0.2 \\
\hline & TOTAL & 15 & 2.6 & 4 & 1.6 & 19 & 2.3 \\
\hline \multirow{14}{*}{ 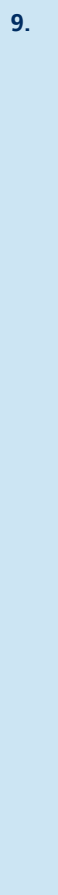 } & Spontaneous preterm & & & & & & \\
\hline & $\begin{array}{l}\text { Intact membranes or membrane rupture less than } \\
24 \text { hours: with chorioamnionitis on placental } \\
\text { histopathology }\end{array}$ & 5 & 0.9 & 32 & 13.0 & 37 & 4.5 \\
\hline & $\begin{array}{l}\text { Intact membranes or membrane rupture less than } \\
24 \text { hours: without chorioamnionitis on placental } \\
\text { histopathology }\end{array}$ & 5 & 0.9 & 13 & 5.3 & 18 & 2.2 \\
\hline & $\begin{array}{l}\text { Intact membranes or membrane rupture less than } \\
24 \text { hours: with clinical evidence of chorioamnionitis, } \\
\text { no examination of placenta }\end{array}$ & 0 & 0.0 & 4 & 1.6 & 4 & 0.5 \\
\hline & $\begin{array}{l}\text { Intact membranes or membrane rupture less than } \\
24 \text { hours: no clinical signs of choriamnionitis, no } \\
\text { examination of placenta }\end{array}$ & 5 & 0.9 & 9 & 3.6 & 14 & 1.7 \\
\hline & $\begin{array}{l}\text { Intact membranes or membrane rupture less than } \\
24 \text { hours: unspecified/unknown placental examination }\end{array}$ & 1 & 0.2 & 0 & 0.0 & 1 & 0.1 \\
\hline & $\begin{array}{l}\text { Membrane rupture } 24 \text { hours or more: with } \\
\text { chorioamnionitis on placental histopathology }\end{array}$ & 14 & 2.4 & 19 & 7.7 & 33 & 4.0 \\
\hline & $\begin{array}{l}\text { Membrane rupture } 24 \text { hours or more: without } \\
\text { chorioamnionitis on placental histopathology }\end{array}$ & 0 & 0.0 & 6 & 2.4 & 6 & 0.7 \\
\hline & $\begin{array}{l}\text { Membrane rupture } 24 \text { hours or more: no clinical signs } \\
\text { of chorioamnionitis, no examination of placenta }\end{array}$ & 4 & 0.7 & 0 & 0.0 & 4 & 0.5 \\
\hline & $\begin{array}{l}\text { Membrane rupture } 24 \text { hours or more: unspecified/ } \\
\text { unknown placental examination }\end{array}$ & 0 & 0.0 & 1 & 0.4 & 1 & 0.1 \\
\hline & $\begin{array}{l}\text { Membrane rupture unknown duration: with } \\
\text { chorioamnionitis on placental histopathology }\end{array}$ & 5 & 0.9 & 3 & 1.2 & 8 & 1.0 \\
\hline & $\begin{array}{l}\text { Membrane rupture unknown duration: without } \\
\text { chorioamnionitis on placental histopathology }\end{array}$ & 0 & 0.0 & 1 & 0.4 & 1 & 0.1 \\
\hline & $\begin{array}{l}\text { Membrane rupture unknown duration: no clinical signs } \\
\text { of chorioamnionitis, no examination of placenta }\end{array}$ & 1 & 0.2 & 6 & 2.4 & 7 & 0.8 \\
\hline & TOTAL & 40 & 6.9 & 94 & 38.1 & 134 & 16.2 \\
\hline \multirow{8}{*}{10.} & Unexplained antepartum death & & & & & & \\
\hline & $\begin{array}{l}\text { With evidence of reduced vascular perfusion on } \\
\text { Doppler studies and / or placental histopathology }\end{array}$ & 37 & 6.4 & 0 & 0.0 & 37 & 4.5 \\
\hline & With chronic villitis & 2 & 0.3 & 0 & 0.0 & 2 & 0.2 \\
\hline & No placental pathology & 87 & 15.0 & 0 & 0.0 & 87 & 10.5 \\
\hline & No examination of placenta & 17 & 2.9 & 0 & 0.0 & 17 & 2.1 \\
\hline & Other specified placental pathology & 40 & 6.9 & 0 & 0.0 & 40 & 4.8 \\
\hline & Unspecified or not known whether placenta examined & 2 & 0.3 & 0 & 0.0 & 2 & 0.2 \\
\hline & TOTAL & 185 & 31.9 & 0 & 0.0 & 185 & 22.4 \\
\hline \multirow{5}{*}{11.} & No obstetric antecedent & & & & & & \\
\hline & Other specified & 0 & 0.0 & 3 & 1.2 & 3 & 0.4 \\
\hline & Accidental asphyxiation & 0 & 0.0 & 1 & 0.4 & 1 & 0.1 \\
\hline & Unknown/unexplained & 0 & 0.0 & 2 & 0.8 & 2 & 0.2 \\
\hline & TOTAL & 0 & 0.0 & 6 & 2.4 & 6 & 0.7 \\
\hline \multicolumn{2}{|c|}{ TOTAL } & 580 & 100.0 & 247 & 100.0 & 827 & 100.0 \\
\hline
\end{tabular}

Source: NSW Maternal and Perinatal Committee, NSW Ministry of Health. 


\section{Causes of perinatal death 2009}

Perinatal deaths were classified according to the PSANZ-PDC, which identifies the single most important factor which led to the chain of events that resulted in the death (Table 112).

\section{Congenital anomaly}

In 2009, congenital anomalies as a group were the most common cause of perinatal death, responsible for 198 deaths. The most common anomalies were chromosomal $(n=48$, $24.2 \%)$. Of these, 15 were trisomy 21, 12 were trisomy 18, 7 were trisomy 13, and 3 were Turner syndrome. Fifty-four deaths were associated with anomalies of the central nervous system (27.3\%) and included 21 deaths due to neural tube defects, 8 deaths associated with congenital hydrocephalus and 7 cases of holoprosencephaly. Twenty-three deaths were associated with anomalies of the cardiovascular system, which included 6 cases of hypoplastic left heart syndrome, 2 cases of common ventricle, and 2 cases of Ebstein's anomaly. Thirtytwo deaths were due to multiple anomalies not associated with a chromosomal anomaly.

\section{Perinatal infection}

Eighty-nine deaths (10.8\%) were due to infection, of which 61 were stillbirths and 28 were neonatal deaths. For 46 deaths there was an associated chorioamnionitis. The most common specified infective organism was group B streptococcus infection $(n=18)$ followed by Escherichia coli $(n=9)$. Four perinatal deaths followed congenital parvovirus infection, two followed a cytomegalovirus infection, and one followed an infection with Listeria Monocytogenes.

\section{Hypertension}

Twenty-nine deaths (3.5\%) were considered to be due to maternal hypertension. There were 22 stillbirths and 7 neonatal deaths. The majority $(n=18)$ occurred in mothers with pre-eclampsia. There were 3 deaths attributed to chronic hypertension, and 4 to gestational hypertension.

\section{Antepartum haemorrhage}

Sixty-seven deaths were due to antepartum haemorrhage, of which 46 were due to placental abruption, 5 were due to placenta praevia, and 1 due to vasa praevia. Of the 46 deaths due to placental abruption, 5 were associated with maternal hypertension.

\section{Maternal disease}

Nineteen deaths were attributed to other maternal conditions including: diabetes $(n=8)$, lupus obstetric syndrome $(n=5)$, and maternal injury $(n=1)$.

\section{Specific perinatal conditions}

Twin-to-twin transfusion accounted for the majority of deaths in this group $(n=16)$, followed by uterine anomaly $(n=12)$, antepartum cord complications $(n=8)$, idiopathic hydrops $(n=6)$, and fetomaternal haemorrhage $(n=6)$.

\section{Hypoxic peripartum death}

There were 26 deaths associated with peripartum hypoxia. Three deaths followed cord prolapse, 2 deaths were associated with shoulder dystocia and 2 deaths followed uterine rupture. Seven deaths occurred before the onset of labour, 4 during labour, 3 at an unspecified time before birth and the remaining 12 deaths occurred in the neonatal period.

\section{Fetal growth restriction}

In 19 cases the main cause of death was considered to be fetal growth restriction (FGR). Of these, 15 were stillbirths and 4 were neonatal deaths. FGR is defined as less than the tenth percentile of birth weight for gestational age with no major congenital anomalies. If a maternal or fetal cause of FGR was known then the cause of death was classified to the underlying cause of the FGR. Stillbirths with evidence of maceration were not classified as FGR unless there was evidence of growth restriction on serial ultrasound during pregnancy or ultrasound prior to fetal demise.

\section{Spontaneous preterm}

There were 134 (16.2\%) perinatal deaths associated with spontaneous preterm birth, which comprises normally formed and appropriately grown babies born before 37 weeks gestation. Of these, 40 (29.9\%) were stillbirths and 94 (70.1\%) were neonatal deaths.

Of all deaths in this category, 73 (54.5\%) were at less than 23 weeks gestation, 59 (44.0\%) were at 23-25 weeks gestation, and 2 (1.5\%) occurred between 26 and 36 weeks gestation. Forty-four deaths (32.8\%) were associated with membrane rupture of 24 hours or more.

\section{Unexplained antepartum death}

Of the 185 unexplained stillbirths, 123 (66.5\%) were low birth weight babies and 130 (70.3\%) were premature. A variety of associated maternal conditions were reported in this group including: multiple pregnancy ( $n=15$ deaths), maternal hypertension ( $n=8)$, diabetes $(n=8)$ and hypothyroidism $(n=2)$. Post-mortem examination was carried out in 64 cases (34.6\%). Placental histopathology results were provided for 163 (88.1\%) unexplained antepartum deaths.

\section{No obstetric antecedent}

No obstetric cause of death was identified for 6 neonatal deaths. Causes included meconium aspiration, massive pulmonary haemorrhage, Sudden Infant Death Syndrome, accidental asphyxiation and kernicterus. 
Table 113. Perinatal deaths by psanz-pdc classification and maternity service level, NSW 2009\#

\begin{tabular}{|c|c|c|c|c|c|c|c|c|c|c|c|c|c|c|}
\hline \multirow{3}{*}{$\begin{array}{l}\text { PSANZ perinatal death } \\
\text { classification }\end{array}$} & \multicolumn{14}{|c|}{ Maternity service level } \\
\hline & \multicolumn{2}{|c|}{ Level 2} & \multicolumn{2}{|c|}{ Level 3} & \multicolumn{2}{|c|}{ Level 4} & \multicolumn{2}{|c|}{ Level 5} & \multicolumn{2}{|c|}{ Level 6} & \multicolumn{2}{|c|}{ Private } & \multicolumn{2}{|c|}{ TOTAL } \\
\hline & No. & $\%$ & No. & $\%$ & No. & $\%$ & No. & $\%$ & No. & $\%$ & No. & $\%$ & No. & $\%$ \\
\hline 1. Congenital anomaly & 0 & 0.0 & 3 & 15.8 & 24 & 16.1 & 31 & 21.5 & 121 & 28.2 & 19 & 22.6 & 198 & 23.9 \\
\hline 2. Perinatal infection & 0 & 0.0 & 4 & 21.1 & 12 & 8.1 & 10 & 6.9 & 45 & 10.5 & 18 & 21.4 & 89 & 10.8 \\
\hline 3. Hypertension & 0 & 0.0 & 0 & 0.0 & 4 & 2.7 & 5 & 3.5 & 18 & 4.2 & 2 & 2.4 & 29 & 3.5 \\
\hline 4. Antepartum haemorrhage & 0 & 0.0 & 2 & 10.5 & 13 & 8.7 & 12 & 8.3 & 33 & 7.7 & 7 & 8.3 & 67 & 8.1 \\
\hline 5. Maternal disease & 0 & 0.0 & 0 & 0.0 & 7 & 4.7 & 1 & 0.7 & 11 & 2.6 & 0 & 0.0 & 19 & 2.3 \\
\hline 6. Specific perinatal conditions & 0 & 0.0 & 1 & 5.3 & 8 & 5.4 & 9 & 6.3 & 32 & 7.5 & 5 & 6.0 & 55 & 6.7 \\
\hline 7. Hypoxic peripartum death & 0 & 0.0 & 1 & 5.3 & 7 & 4.7 & 7 & 4.9 & 8 & 1.9 & 2 & 2.4 & 26 & 3.1 \\
\hline 8. Fetal growth restriction & 1 & 100.0 & 0 & 0.0 & 1 & 0.7 & 2 & 1.4 & 14 & 3.3 & 1 & 1.2 & 19 & 2.3 \\
\hline 9. Spontaneous preterm & 0 & 0.0 & 1 & 5.3 & 22 & 14.8 & 27 & 18.8 & 75 & 17.5 & 9 & 10.7 & 134 & 16.2 \\
\hline 10. Unexplained antepartum death & 0 & 0.0 & 7 & 36.8 & 51 & 34.2 & 38 & 26.4 & 69 & 16.1 & 20 & 23.8 & 185 & 22.4 \\
\hline 11. No obstetric antecedent & 0 & 0.0 & 0 & 0.0 & 0 & 0.0 & 2 & 1.4 & 3 & 0.7 & 1 & 1.2 & 6 & 0.7 \\
\hline TOTAL & 1 & 100.0 & 19 & 100.0 & 149 & 100.0 & 144 & 100.0 & 429 & 100.0 & 84 & 100.0 & 827 & 100.0 \\
\hline
\end{tabular}

\section{Obstetric cause of perinatal} death by hospital service level

Maternity service levels are described in the Explanatory Notes of the Methods section (page 13). The majority of perinatal deaths occurred in level 6 hospitals (48.7\%, Table 113). The proportion of unexplained intrauterine deaths was substantially lower in level 6 hospitals than other hospitals, possibly due to better access to perinatal postmortem services. The proportion of deaths associated with congenital abnormalities was highest in level 6 hospitals, reflecting patterns of referral for diagnosis and treatment.

\section{Time of death}

Of the 827 perinatal deaths reviewed for 2009, 378 (45.7\%) occurred before the onset of labour, 83 (10.0\%) occurred during labour, 119 (14.4\%) occurred at an unspecified time before birth, and 247 (29.9\%) were neonatal deaths.

Of the 83 deaths reported to have occurred during labour, 55 (66.3\%) occurred at less than 23 weeks gestation, 13 (15.7\%) occurred at 23 to 25 weeks gestation, and 15 (18.1\%) occurred at 26 weeks gestation or more.

\section{Perinatal deaths associated with maternal drug dependency-abuse}

Three perinatal deaths were directly attributed to maternal drug dependency or drug abuse in 2009. A further nine deaths occurred among mothers who had a history of drug dependency or abuse, but drug use was not considered to be the main cause of death.

\section{Postmortem examination}

Postmortem examination is valuable in ascertaining or confirming the cause of death, identifying additional factors that may have contributed to the death, and counselling parents about the cause of death. Postmortem examinations were carried out for 249 (30.1\%) perinatal deaths: 204 stillborn infants (35.2\% of all reported stillbirths) and 45 neonatal deaths (18.2\% of all reported neonatal deaths). Placental histopathology was carried out for 690 (83.4\%) perinatal deaths.

\section{Causes of neonatal death 2009}

In 2009 extreme prematurity (26 weeks gestation or less) accounted for $51.4 \%$ of all neonatal deaths (Table 114). Congenital anomalies were the next most common cause of neonatal death, accounting for about one in five deaths.

Of the 247 neonatal deaths, 218 (88.3\%) were preterm (Table 115). Twenty-nine neonatal deaths were among babies born at term, of which 11 deaths were due to neurological conditions, and a further 10 deaths were due to congenital anomalies.

\section{References}

1. NSW Ministry of Health. Hospital procedures for Review and Reporting of Perinatal Deaths. Available at www. health.nsw.gov.au/policies/pd/2006/PD2006_006.html.

2. Perinatal Society of Australia and New Zealand. Clinical Practice Guideline for Perinatal Mortality Audit. PSANZ, 2005 
Table 114. Neonatal deaths by PSANZ-NDC classification, NSW 2006-2009\#

\begin{tabular}{|c|c|c|c|c|c|c|c|c|}
\hline \multirow[t]{3}{*}{ PSANZ neonatal death classification } & \multicolumn{8}{|c|}{ Year } \\
\hline & \multicolumn{2}{|c|}{2006} & \multicolumn{2}{|c|}{2007} & \multicolumn{2}{|c|}{2008} & \multicolumn{2}{|c|}{2009} \\
\hline & No. & $\%$ & No. & $\%$ & No. & $\%$ & No. & $\%$ \\
\hline \multicolumn{9}{|l|}{ Congenital abnormality } \\
\hline Central nervous system & 5 & 1.9 & 20 & 7.7 & 18 & 6.7 & 11 & 4.5 \\
\hline Cardiovascular system & 8 & 3.0 & 10 & 3.9 & 4 & 1.5 & 5 & 2.0 \\
\hline Urinary system & 7 & 2.6 & 6 & 2.3 & 3 & 1.1 & 4 & 1.6 \\
\hline Gastrointestinal system & 5 & 1.9 & 0 & 0.0 & 2 & 0.7 & 1 & 0.4 \\
\hline Chromosomal & 16 & 5.9 & 4 & 1.5 & 9 & 3.3 & 9 & 3.6 \\
\hline Metabolic & 2 & 0.7 & 2 & 0.8 & 1 & 0.4 & 0 & 0.0 \\
\hline Multiple & 12 & 4.5 & 6 & 2.3 & 12 & 4.5 & 8 & 3.2 \\
\hline Musculoskeletal & 4 & 1.5 & 4 & 1.5 & 5 & 1.9 & 3 & 1.2 \\
\hline Respiratory & 1 & 0.4 & 1 & 0.4 & 1 & 0.4 & 2 & 0.8 \\
\hline Diaphragmatic hernia & 3 & 1.1 & 5 & 1.9 & 1 & 0.4 & 2 & 0.8 \\
\hline Haematological & 0 & 0.0 & 1 & 0.4 & 0 & 0.0 & 0 & 0.0 \\
\hline Tumours & 1 & 0.4 & 2 & 0.8 & 0 & 0.0 & 0 & 0.0 \\
\hline Other specified congenital abnormality & 1 & 0.4 & 1 & 0.4 & 3 & 1.1 & 1 & 0.4 \\
\hline Unspecified & 1 & 0.4 & 1 & 0.4 & 0 & 0.0 & 0 & 0.0 \\
\hline TOTAL & 66 & 24.5 & 63 & 24.3 & 59 & 21.9 & 46 & 18.6 \\
\hline \multicolumn{9}{|l|}{ Extreme prematurity } \\
\hline Not resuscitated & 72 & 26.8 & 92 & 35.5 & 96 & 35.7 & 112 & 45.3 \\
\hline Unsuccessful resuscitation & 10 & 3.7 & 11 & 4.2 & 12 & 4.5 & 5 & 2.0 \\
\hline Resuscitation unspecified or unknown & 26 & 9.7 & 17 & 6.6 & 20 & 7.4 & 10 & 4.0 \\
\hline TOTAL & 108 & 40.1 & 120 & 46.3 & 128 & 47.6 & 127 & 51.4 \\
\hline \multicolumn{9}{|l|}{ Cardio-respiratory disorders } \\
\hline Hyaline membrane disease / Respiratory distress syndrome & 18 & 6.7 & 5 & 1.9 & 11 & 4.1 & 6 & 2.4 \\
\hline Meconium aspiration syndrome & 4 & 1.5 & 2 & 0.8 & 2 & 0.7 & 1 & 0.4 \\
\hline Primary persistent pulmonary hypertension & 1 & 0.4 & 1 & 0.4 & 1 & 0.4 & 2 & 0.8 \\
\hline Pulmonary hypoplasia & 4 & 1.5 & 3 & 1.2 & 4 & 1.5 & 5 & 2.0 \\
\hline Chronic neonatal lung disease & 0 & 0.0 & 0 & 0.0 & 1 & 0.4 & 0 & 0.0 \\
\hline Other & 3 & 1.1 & 5 & 1.9 & 2 & 0.7 & 2 & 0.8 \\
\hline TOTAL & 30 & 11.2 & 16 & 6.2 & 21 & 7.8 & 16 & 6.5 \\
\hline \multicolumn{9}{|l|}{ Infection } \\
\hline Congenital bacterial & 5 & 1.9 & 6 & 2.3 & 14 & 5.2 & 5 & 2.0 \\
\hline Acquired bacterial & 6 & 2.2 & 2 & 0.8 & 3 & 1.1 & 2 & 0.8 \\
\hline Congenital viral & 2 & 0.7 & 1 & 0.4 & 0 & 0.0 & 0 & 0.0 \\
\hline Acquired viral & 0 & 0.0 & 1 & 0.4 & 0 & 0.0 & 0 & 0.0 \\
\hline Protozoal eg Toxoplasma & 0 & 0.0 & 0 & 0.0 & 0 & 0.0 & 3 & 1.2 \\
\hline Other & 0 & 0.0 & 1 & 0.4 & 1 & 0.4 & 0 & 0.0 \\
\hline Unspecified organism & 5 & 1.9 & 1 & 0.4 & 2 & 0.7 & 1 & 0.4 \\
\hline TOTAL & 18 & 6.7 & 12 & 4.6 & 20 & 7.4 & 11 & 4.5 \\
\hline \multicolumn{9}{|l|}{ Neurological } \\
\hline Hypoxic ischaemic encephalopathy / perinatal asphyxia & 27 & 10.0 & 27 & 10.4 & 14 & 5.2 & 26 & 10.5 \\
\hline Intracranial haemorrhage & 8 & 3.0 & 10 & 3.9 & 15 & 5.6 & 13 & 5.3 \\
\hline Other & 0 & 0.0 & 1 & 0.4 & 0 & 0.0 & 1 & 0.4 \\
\hline TOTAL & 35 & 13.0 & 38 & 14.7 & 29 & 10.8 & 40 & 16.2 \\
\hline \multicolumn{9}{|l|}{ Gastrointestinal } \\
\hline Necrotising enterocolitis & 3 & 1.1 & 2 & 0.8 & 5 & 1.9 & 1 & 0.4 \\
\hline TOTAL & 3 & 1.1 & 2 & 0.8 & 5 & 1.9 & 1 & 0.4 \\
\hline \multicolumn{9}{|l|}{ Other } \\
\hline SIDS & 1 & 0.4 & & 0 & 0.0 & 0 & 0.0 & 0 \\
\hline Unclassified Sudden Infant Death & 0 & 0.0 & 1 & 0.4 & 1 & 0.4 & 0 & 0.0 \\
\hline Possible SIDS & 0 & 0.0 & 0 & 0.0 & 1 & 0.4 & 0 & 0.0 \\
\hline Multisystem failure & 0 & 0.0 & 0 & 0.0 & 0 & 0.0 & 1 & 0.4 \\
\hline Trauma & 1 & 0.4 & 0 & 0.0 & 0 & 0.0 & 0 & 0.0 \\
\hline Other specified & 3 & 1.1 & 0 & 0.0 & 3 & 1.1 & 2 & 0.8 \\
\hline Unknown/Undetermined & 4 & 1.5 & 7 & 2.7 & 2 & 0.7 & 3 & 1.2 \\
\hline TOTAL & 9 & 3.3 & 8 & 3.1 & 7 & 2.6 & 6 & 2.4 \\
\hline TOTAL & 269 & 100.0 & 259 & 100.0 & 269 & 100.0 & 247 & 100.0 \\
\hline
\end{tabular}

Source: NSW Maternal and Perinatal Committee, NSW Ministry of Health.

\#Figures may differ from previous reports due to additional information being received after publication. 
Table 115. Neonatal deaths by PSANZ-NDC classification and gestational age, NSW 2009

\begin{tabular}{|c|c|c|c|c|c|c|}
\hline \multirow[t]{3}{*}{ PSANZ neonatal death classification } & \multicolumn{6}{|c|}{ Gestational age } \\
\hline & \multicolumn{2}{|c|}{$<37$ weeks } & \multicolumn{2}{|c|}{ > 37 weeks } & \multicolumn{2}{|c|}{ TOTAL } \\
\hline & No. & $\%$ & No. & $\%$ & No. & $\%$ \\
\hline \multicolumn{7}{|l|}{ Congenital abnormality } \\
\hline Central nervous system & 8 & 3.7 & 3 & 10.3 & 11 & 4.5 \\
\hline Cardiovascular system & 4 & 1.8 & 1 & 3.4 & 5 & 2.0 \\
\hline Urinary tract & 3 & 1.4 & 1 & 3.4 & 4 & 1.6 \\
\hline Gastrointestinal tract & 1 & 0.5 & 0 & 0.0 & 1 & 0.4 \\
\hline Chromosomal & 7 & 3.2 & 2 & 6.9 & 9 & 3.6 \\
\hline Multiple & 6 & 2.8 & 2 & 6.9 & 8 & 3.2 \\
\hline Musculoskeletal & 3 & 1.4 & 0 & 0.0 & 3 & 1.2 \\
\hline Respiratory & 2 & 0.9 & 0 & 0.0 & 2 & 0.8 \\
\hline Diaphragmatic hernia & 1 & 0.5 & 1 & 3.4 & 2 & 0.8 \\
\hline Other specified congenital abnormality & 1 & 0.5 & 0 & 0.0 & 1 & 0.4 \\
\hline TOTAL & 36 & 16.5 & 10 & 34.5 & 46 & 18.6 \\
\hline \multicolumn{7}{|l|}{ Extreme prematurity } \\
\hline Not resuscitated & 112 & 51.4 & 0 & 0.0 & 112 & 45.3 \\
\hline Unsuccessful resuscitation & 5 & 2.3 & 0 & 0.0 & 5 & 2.0 \\
\hline Resuscitation unspecified or unknown & 10 & 4.6 & 0 & 0.0 & 10 & 4.0 \\
\hline TOTAL & 127 & 58.3 & 0 & 0.0 & 127 & 51.4 \\
\hline \multicolumn{7}{|l|}{ Cardio-respiratory disorders } \\
\hline Hyaline membrane disease / Respiratory distress syndrome & 6 & 2.8 & 0 & 0.0 & 6 & 2.4 \\
\hline Meconium aspiration syndrome & 0 & 0.0 & 1 & 3.4 & 1 & 0.4 \\
\hline Primary persistent pulmonary hypertension & 2 & 0.9 & 0 & 0.0 & 2 & 0.8 \\
\hline Pulmonary hypoplasia & 5 & 2.3 & 0 & 0.0 & 5 & 2.0 \\
\hline Other & 1 & 0.5 & 1 & 3.4 & 2 & 0.8 \\
\hline TOTAL & 14 & 6.4 & 2 & 6.9 & 16 & 6.5 \\
\hline \multicolumn{7}{|l|}{ Infection } \\
\hline Congenital bacterial & 3 & 1.4 & 2 & 6.9 & 5 & 2.0 \\
\hline Acquired bacterial & 1 & 0.5 & 1 & 3.4 & 2 & 0.8 \\
\hline Protozoal eg Toxoplasma & 3 & 1.4 & 0 & 0.0 & 3 & 1.2 \\
\hline Unspecified organism & 1 & 0.5 & 0 & 0.0 & 1 & 0.4 \\
\hline TOTAL & 8 & 3.7 & 3 & 10.3 & 11 & 4.5 \\
\hline \multicolumn{7}{|l|}{ Neurological } \\
\hline Hypoxic ischaemic encephalopathy / perinatal asphyxia & 16 & 7.3 & 10 & 34.5 & 26 & 10.5 \\
\hline Intracranial haemorrhage & 13 & 6.0 & 0 & 0.0 & 13 & 5.3 \\
\hline Other & 0 & 0.0 & 1 & 3.4 & 1 & 0.4 \\
\hline TOTAL & 29 & 13.3 & 11 & 37.9 & 40 & 16.2 \\
\hline \multicolumn{7}{|l|}{ Gastrointestinal } \\
\hline Necrotising enterocolitis & 1 & 0.5 & 0 & 0.0 & 1 & 0.4 \\
\hline TOTAL & 1 & 0.5 & 0 & 0.0 & 1 & 0.4 \\
\hline \multicolumn{7}{|l|}{ Other } \\
\hline Multisystem failure & 1 & 0.5 & 0 & 0.0 & 1 & 0.4 \\
\hline Other specified & 1 & 0.5 & 1 & 3.4 & 2 & 0.8 \\
\hline Unknown/Undetermined & 1 & 0.5 & 2 & 6.9 & 3 & 1.2 \\
\hline TOTAL & 3 & 1.4 & 3 & 10.3 & 6 & 2.4 \\
\hline TOTAL & 218 & 100.0 & 29 & 100.0 & 247 & 100.0 \\
\hline
\end{tabular}

Source: NSW Maternal and Perinatal Committee, NSW Ministry of Health. 